Observations de la comète 1886 VII (Finlay) faites à l'Observatoire de Tachkent.

\begin{tabular}{|c|c|c|c|c|c|c|c|c|c|c|}
\hline 1886 & T.M.Tachk. & $\Delta \alpha$ & $\Delta \delta$ & $\mathrm{Cp}$. & $\alpha$ app. & $\log p \cdot \Delta$ & $\delta$ app. & $\log p \cdot \Delta$ & Red. ad 1. app. & \\
\hline t. 28 & $7^{\mathrm{h}}$ I $2^{\mathrm{m}} 37^{\mathrm{s}}$ & $+0^{m} 4^{\mathrm{s}} .0 \mathrm{x}$ & $+6^{\prime} 3$ & 7 & I $8^{\mathrm{h}} 40^{\mathrm{m}} 12^{\mathrm{s}} \cdot 59$ & & $-26^{\circ}$ I $3^{\prime} 211^{\prime \prime} 2$ & 3 & +1 s.68 & \\
\hline 29 & 64242 & +349.87 & +1050.2 & 7 & $\begin{array}{lll}8 & 43 & 58.44\end{array}$ & 9 . & $-26 \quad 9$ & .867 & $+1.67+8.6$ & \\
\hline ov. 18 & $\begin{array}{lll}7 & 19 & 2\end{array}$ & $-2 \quad 27.65$ & -15 I 7.6 & 7 & 2010 & $9.53^{\circ}$ & -2244 & $0.85^{6}$ & $+1.71+12.0$ & \\
\hline
\end{tabular}

Positions moyennes des Étoiles de Comparaison.

\begin{tabular}{|c|c|c|c|}
\hline * & $\alpha 1886.0$ & $\delta$ I 886.0 & Autorité \\
\hline 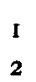 & 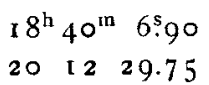 & $\begin{array}{llr}-26^{\circ} & 20^{\prime} & 1.3 \\
-22 & 28 & 54.6\end{array}$ & $\begin{array}{l}1 / 2\left(\mathrm{AOe}_{2}{ }^{1} 865^{2}+\mathrm{Lam}_{6} \text { I }_{3} \mathrm{I}\right) \\
1 / 2\left(\mathrm{AOe}_{2} 203^{8} 9+\mathrm{Lam}_{6} 488\right)\end{array}$ \\
\hline
\end{tabular}

Tachkent 1887 Février $\boldsymbol{z}$.

H. Pomerantzeff.

\title{
Beobachtungen von Cometen
}

angestellt von Herrn B. von Engelhardt am Fadenmikrometer des rainch. Aequatoreals seiner Privatsternwarte in Dresden.

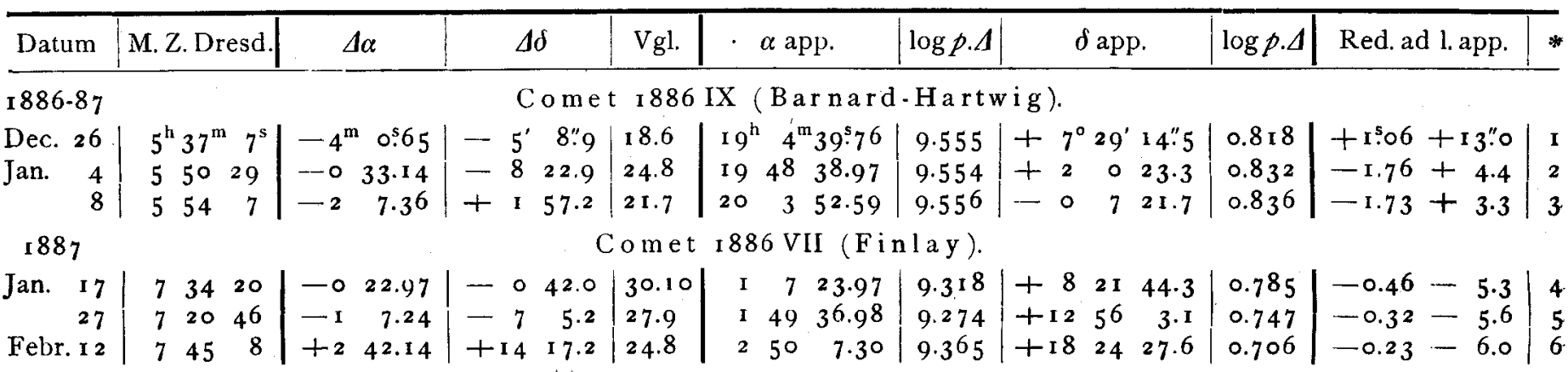

Mittlere Oerter der Vergleichsterne für den Anfang des Beobachtungsjahres.

\begin{tabular}{|c|c|c|c|}
\hline * & $\alpha$ (Wolfers) & $\delta$ (Auwers) & Autorität \\
\hline 1 & $19^{\mathrm{h}} \quad 8^{\mathrm{m}} 39^{\mathrm{s}} \cdot 35$ & $+7^{\circ} 34^{\prime}$ I0." 4 & 2397 \\
\hline 2 & $\begin{array}{lll}19 & 49 & 13.87\end{array}$ & 841.8 & $1 / 2\left(W_{1}\right.$ I $_{206}+\operatorname{Lam}_{1} 7$ I 57$)$ \\
\hline 3 & I. 68 & 9.22 .2 & Gött. 5534.35 \\
\hline
\end{tabular}

\begin{tabular}{|c|c|c|c|}
\hline$*$ & $\alpha$ (Wolfers) & $\delta$ (Auwers) & Autorität \\
\hline 4 & $\mathrm{I}^{\mathrm{h}} \quad 7^{\mathrm{m}} 47^{\mathrm{s}}: 40$ & $+8^{\circ} 22^{\prime} 33^{\prime \prime} 6$ & $1 / 2\left(\mathrm{Sj} .39^{2}+\right.$ Gl. 293$)$ \\
\hline 5 & I $5 \circ \quad 44.54$ & $\begin{array}{ll}3 & 13.9\end{array}$ & $R \ddot{u}_{2} 99 \mathrm{I}$ \\
\hline 6 & $\begin{array}{lll}2 & 47 & 25.39\end{array}$ & +181016.4 & A. N. I I I. I 67 \\
\hline
\end{tabular}

Vergleichung der Beobachtungen des Cometen r 886 VII (Finlay) mit der Ephemeride von Herrn H. Kloock (B-R):

\begin{tabular}{c|c|c|c}
\hline \multicolumn{1}{c|}{1887} & $\Delta \alpha$ & $\Delta \delta$ & Ephemeride \\
\hline Jan. 17 & -4.99 & -26.2 & A. N. 2765 \\
27 & -6.54 & -28.8 & \\
Febr. 1 2 & +2.29 & +23.6 & A. N. 2769
\end{tabular}

Bemerkungen.

Comet I 886 IX (Barnard-Hartwig).

Dec. 26. Der Kern sieht aus wie ein sehr helles Scheibchen von bläulich-weisser Farbe und ist in beleuchtetem Gesichtsfelde "gut sichtbar. Seine Nebelhülle hat 2' Durchmesser. Der Schweif ist gekrümmt, die convexe Seite geht voran. Der Stern $7^{\mathrm{m}} \cdot 2 \mathrm{DM}$. $+7^{\circ} \cdot 3987$ stand um $0^{\mathrm{h}} \cdot 3$ Sternzeit ungefähr in der Schweifaxe, und der Schweif erstreckte sich in nördlicher Richtung noch etwa I $0^{\prime}$ über diesen Stern hinaus.

Jan. 4. Luft gut, heller Mondschein und helle Abenddämmerung. - - Im $5 \mathrm{z}$. Sucher auf den ersten.Blick gesehen, als noch kein Stern sichtbar war.

8. Ein 'Tag vor dem Vollmond. Dunst. - Ziemlich hell, aber sehr verwaschen. Durchmesser o:5. 